\title{
Travel distance to flu and COVID-19 vaccination sites for people with disabilities and age 65 and older, Chicago metropolitan area
}

\author{
Aida Rosalia Guhlincozzi \\ Geography and Geographic Information Science Department, \\ University of Illinois at Urbana, Champaign, Illinois, USA, and \\ Aynaz Lotfata \\ Geography Department, Chicago State University, Chicago, Illinois, USA
}

Flu and

COVID-19

vaccination

sites

\begin{abstract}
Purpose - Having easy access to the flu and COVID-19 vaccination sites may be important for controlling the spread of the infection. Chicago implemented a broad strategy of opening COVID-19 vaccination locations across the city in a variety of locations.

Design/methodology/approach - We defined access as having vaccinations within one mile. Data came from the American Community Survey (ACS), Centers for Disease Control and Prevention (CDC), Social Vulnerability Index (SVI), Illinois Department of Public Health (IDPH) and the Chicago Data Portal. We calculated the street-network distance from the population-weighted centroid of census tracts to the nearest vaccination sites before, during and post COVID-19 pandemic. We compared the demographics of census tracts within one mile to those greater than one mile during each period.

Findings - People age 65 and above and with disabilities saw significant decreases in flu vaccination site access to locations within one mile of their census tract in 2020-2021 compared to 2018-2019. The COVID-19 vaccination sites mimic these flu vaccination site patterns, suggesting a severe lack of geographic access for a group likely to experience limited mobility. Results combining instances of both flu and COVID-19 vaccination sites suggest that making COVID-19 vaccination sites available at flu shot site locations would significantly reduce the number of people with limited mobility lacking geographic access.

Originality/value - Policymakers should explore how this expanded network of vaccination locations could facilitate permanent improvements to access to vaccination sites for people with disabilities.
\end{abstract}

Keywords People with disability, Elderly, COVID-19 vaccination, Accessibility, United States

Paper type Short report

\section{Introduction}

In the United States, as of 2018, approximately 61 million people live with disabilities.

As of 2016, approximately 49.2 million people in the United States are over the age of 65 . Of the group that is over the age of 65, 2 out of 5 people over the age of 65 are likely to have some type of disability. Meanwhile, of the 61 million people living with a disability, $13 \%$ of them likely have a mobility-related disability $[1,2]$.

When trying to access healthcare, these groups are then at a higher disadvantage, facing multiple barriers. For people older than 65 , a number of both spatial and psychological

(C) Aida Rosalia Guhlincozzi and Aynaz Lotfata. Published in Journal of Health Research. Published by Emerald Publishing Limited. This article is published under the Creative Commons Attribution (CC BY 4.0) licence. Anyone may reproduce, distribute, translate and create derivative works of this article (for both commercial and non-commercial purposes), subject to full attribution to the original publication and authors. The full terms of this licence may be seen at http://creativecommons.org/licences/by/4.0/ legalcode

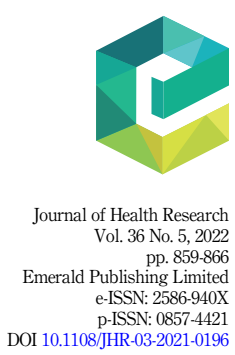


JHR

36,5

860

barriers are at play. Transportation and cost were notable but also issues with poor interactions with the doctor and a lack of support from the physician. Feeling unsupported by the physician, a lack of trust in the physician lead to the elderly patient feeling uncomfortable and unable to visit the doctor [3].

For people with disabilities, they face poverty at a rate that is more than double that of people without disabilities, at $29 \%$ in 2018 . Access to private health insurance is limited by half - only $44 \%$ for people with disabilities, while $89 \%$ of people with disabilities are covered by public health insurance [4].

Access to pharmaceuticals is difficult for both people over the age of 65 and people with disabilities often because of cost. Geographically, the cost is a varying factor throughout the country, and states face complicated policymaking to maintain cost-subsidizing programs as federal healthcare policies shift. People with disabilities are also forced to navigate these complex cost-subsidizing programs as well [5]. In the wake of the COVID-19 pandemic, people with disabilities and those over the age of 65 who need caretakers or assistance from other people, have faced physical access issues to healthcare settings because of requirements to reduce the number of additional people allowed in a healthcare space [6]. These experiences, in Chicago specifically, are likely to be worse for people with disabilities or over the age of 65 and also from a racial or ethnic minoritized group, given the disparity of pharmacy access in Chicago [7].

Understanding how these disparities in care impact vaccine distribution in heavily populated areas is crucial to preparing a successful vaccination roll-out plan for COVID-19 and other future potential diseases, including both the flu and booster shots for COVID-19 variants. This study provides insight into access for particularly vulnerable groups in Chicago and makes recommendations for improving access. The purposes of this study were to compare access to the flu and COVID-19 vaccination sites before, during and post COVID-19 for people with disabilities and age 65 and older and to use publicly available data to propose the most impactful flu site to make permanent vaccination locations.

\section{Methods}

Data for this study was obtained from the Illinois Department of Public Health (IDPH) [8], the Chicago Data Portal [9], American Community Survey (ACS) Centers of Population by Census Tract [10] and Centers for Disease Control and Prevention (CDC) 2018 Social Vulnerability Index (SVI) [11]. The CDC 2018 Social Vulnerability Index quantifies social vulnerability to hazards like natural disasters or disease epidemics. The SVI is composed of four themes: (1) Socioeconomic Status, (2) Household Composition, (3) Race, Ethnicity, Language and (4) Housing, Transportation. The SVI data was used to estimate the number and percentage of people with disabilities and age 65 and older per Census Tract.

Three sets of vaccination sites were analyzed: before COVID-19, the 287 2018-2019 flu shot locations, during COVID-19, 177 COVID-19 vaccination sites, and post-COVID-19, 341 potential vaccination locations (using 177 COVID-19 vaccination sites and 164 2020-2021 flu vaccination sites).

Health centers and vaccinations sites are basic urban services in the current pandemic situation. They should be in good accessibility for people with disabilities and age 65 and above at a greater risk of infection and dying with poorer health. The existing studies [12] found a one-mile travel distance contributes to decreasing social and physical discomforts in access to the vaccination sites and healthcare during the pandemics. Population Weighted Centroid, and before, during and post COVID-19 flu and COVID-19 vaccination sites were used to estimate the testing sites within one mile of the population with disabilities and age 65 and older per Census tract spatial unit. The street-based Closest Facility network analysis 
was used in the ArcGIS Pro to examine the variations in the population with disabilities within one-mile driving distance of the flu and COVID-19 vaccinations sites before, during and post COVID-19. We summarized the drive distances by population with disabilities, age 65 and older, access to the private vehicle, people with health insurance and poverty percentages within one mile of the vaccination sites.

Flu and

COVID-19

vaccination

sites

\section{Ethical consideration}

This study used the public-use data, and it does not include IRB confirmation.

\section{Results}

Before the COVID-19 Pandemic, of the 335,707 people age 65 and over, 253,827 (75.6\%) had been within one-mile driving distance to flu vaccination sites. Meanwhile, 81,880 (24.4\%) of people age 65 and older had been over a mile driving distance to flu vaccination sites. Further, of the 292,721 uninsured people, 232,371 (79.4.1\%) and of the 329,474 people without vehicle, $227,500(80 \%)$ had been within a mile driving distance to flu vaccination sites (Figure $1 \mathrm{~A}$ and Table 1).

During the COVID-19 pandemic, of the 335,683 people age 65 and over, 228,648 (68.11\%) had been within one-mile driving distance to flu vaccination sites. Meanwhile, 107,035 $(31.9 \%)$ of people age 65 and older had been over a mile driving distance to flu vaccination sites. Further, of the 467,778 uninsured people, 174,516 (59.5\%) and of the 288,045 people without vehicle, $186,910(65.6 \%)$ had been within a mile driving distance to flu vaccination sites (Figure $1 \mathrm{~B}$ and Table 1).

During the COVID-19 pandemic, of the 339,104 people age 65 and older, 174,945 (51.6\%) had been within one-mile driving distance to the COVID-19 vaccination sites. Meanwhile, $164,159(48.4 \%)$ of people age 65 and older had been over a mile driving distance to flu vaccination sites (Figure $1 \mathrm{C}$ and Table 1 ).

In a post-COVID-19 pandemic using both COVID-19 vaccination sites and flu vaccination sites, of the 335,838 people age 65 and older, $65,252,154(75.1 \%)$ had been within one-mile driving distance to flu vaccination sites. Meanwhile, 83,684 (24.9\%) of people age 65 and older had been over a mile driving distance to flu vaccination sites. Further, of the 293,191 uninsured people, 234,004 (79.8.5\%) and of the 284,310 people without vehicle, 235,071 $(82.7 \%)$ had been within a mile driving distance to flu vaccination sites (Figure 1D and Table 1).

Before the COVID-19 pandemic, of the 285,435 people with disabilities, 210,957 (73.9\%) of the disabled people had been within one -mile driving distance to flu vaccination sites. Meanwhile, $74,478(26.09 \%)$ of people with disabilities had been over a mile driving distance to flu vaccination sites (Figure $2 \mathrm{~A}$ and Table 1 ).

During the COVID-19 pandemic, of the 285,424 people with disabilities, 190,364 (66.7\%) of disabled people had been within one -mile driving distance from the flu vaccination sites. For those over a mile driving distance, the number of people with disabilities was 95,060 (33.3\%) (Figure 2B and Table 1).

For access to COVID-19 vaccinations during the COVID-19 pandemic, of the 287,027 people with disabilities, 155,638 (54.2\%) were within one mile of COVID-19 vaccination locations. For those over a mile away from COVID-19 vaccination sites, there were 131,389 people $(45.8 \%$ ) with disabilities (Figure $2 \mathrm{C}$ and Table 1 ).

In a post-COVID-19 pandemic using both COVID-19 vaccination sites and flu vaccination sites, of the 285,695 people with disabilities, 219,424 (76.8\%) of disabled people are within one -mile driving distance from potential COVID-19 vaccination sites. 66,271 people with disabilities $(23.2 \%)$ are over a mile driving distance to the sites (Figure 2D and Table 1 ). 
JHR
36,5

\section{2}

Figure 1.

Vaccination sites for people over the age of 65 by driving distance to nearest vaccination site. A: Estimate of people over the age of 65 to flu vaccination sites in 2018-2019. B: Estimate of people over the age of 65 to flu vaccination sites in 2020-2021. C: Estimate of people over the age of 65 to COVID-19 vaccination sites in 2020-2021. D: Estimate of people over the age of 65 to COVID-19 and flu vaccination sites in 2020-2021

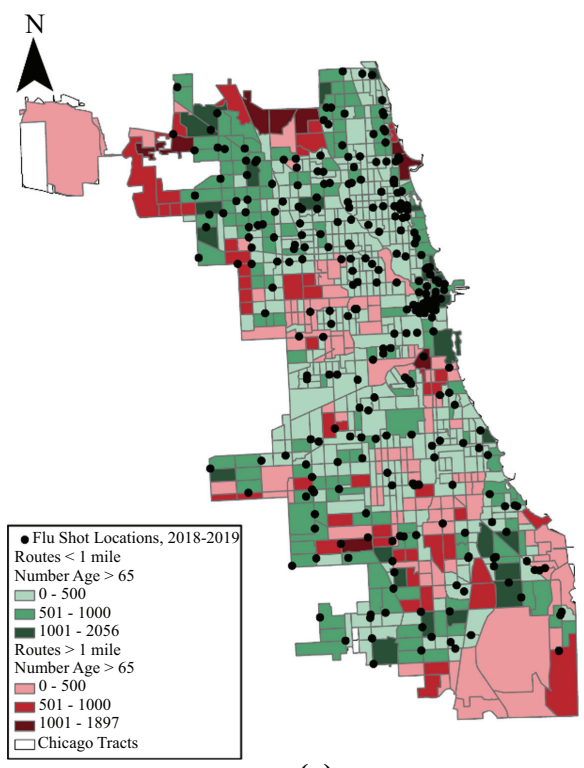

(a)

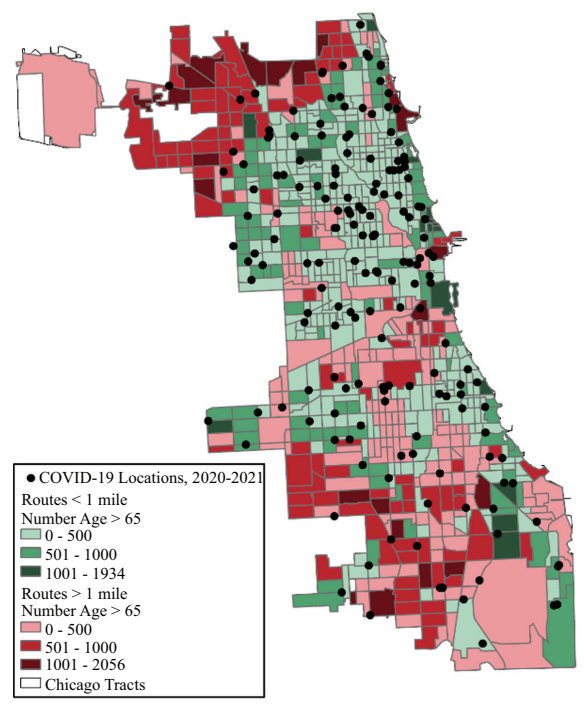

(c)

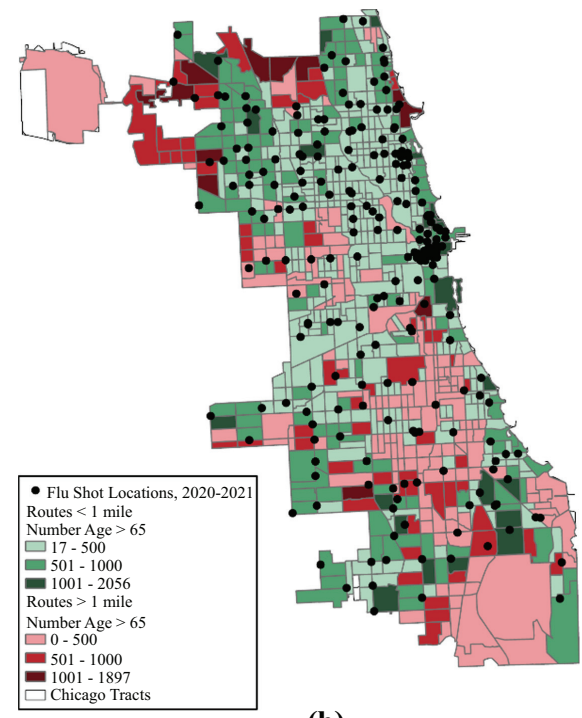

(b)

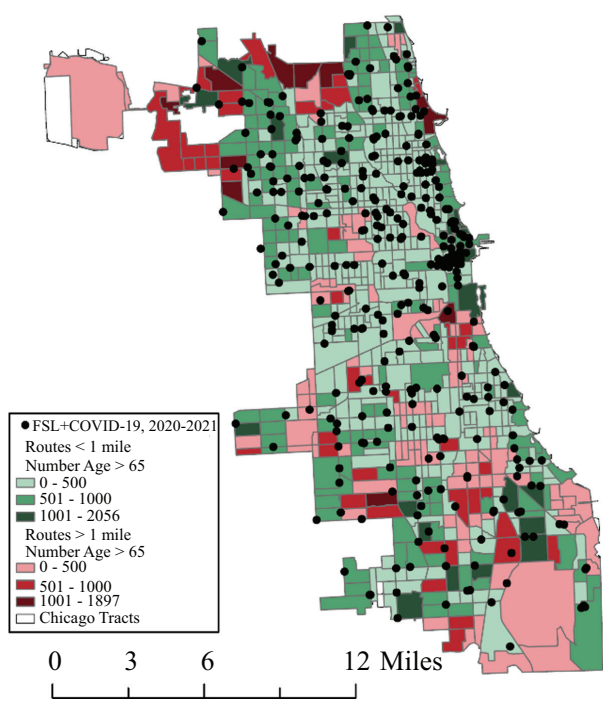

(d)

Source(s): Data from ACS 2018, CDC Social Vulnerability Index 2018, and Illinois Department of Public Health

\section{Discussion}

Figure 1A shows that from 2018 to 2019, geographic flu vaccination access worsened for people over the age of 65, particularly throughout south/central Chicago. Figure 1C's display of current COVID-19 vaccination sites, as of February 2021, suggests poor access, with only 


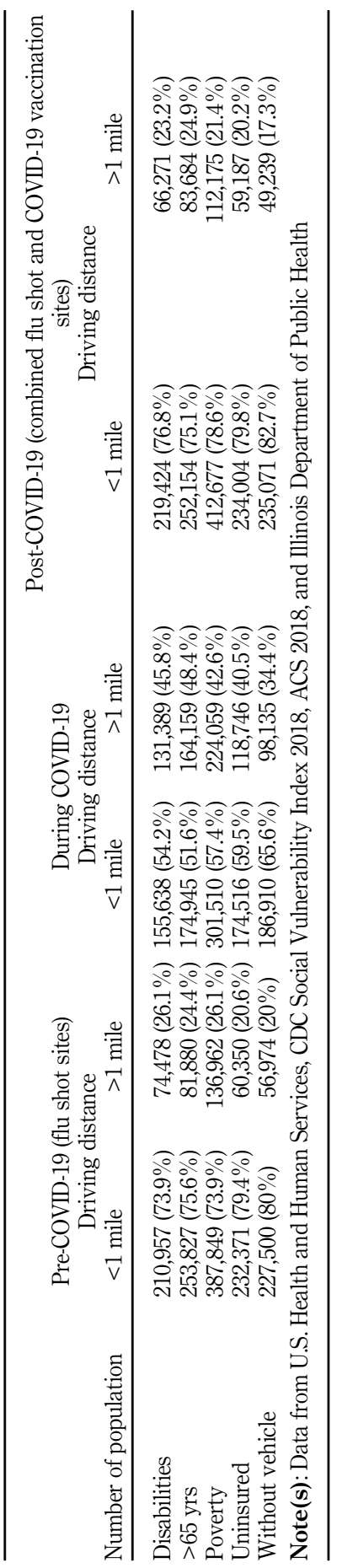

Flu and

COVID-19

vaccination

sites

863

Table 1.

Percent of vulnerable populations less than and greater than one mile away from flu, COVID-19 vaccination sites in 2018-2019 and 2020-2021 
JHR
36,5

\section{4}

Figure 2.

Vaccination sites for disabled people by driving distance to nearest vaccination site. A: Estimate of disabled people to flu vaccination sites in 2018-2019. B: Estimate of disabled people to flu vaccination sites in 2020-2021. C: Estimate of disabled people to COVID-19 vaccination sites in 2020-2021. D: Estimate of disabled people to COVID-19 and flu vaccination sites in 2020-2021

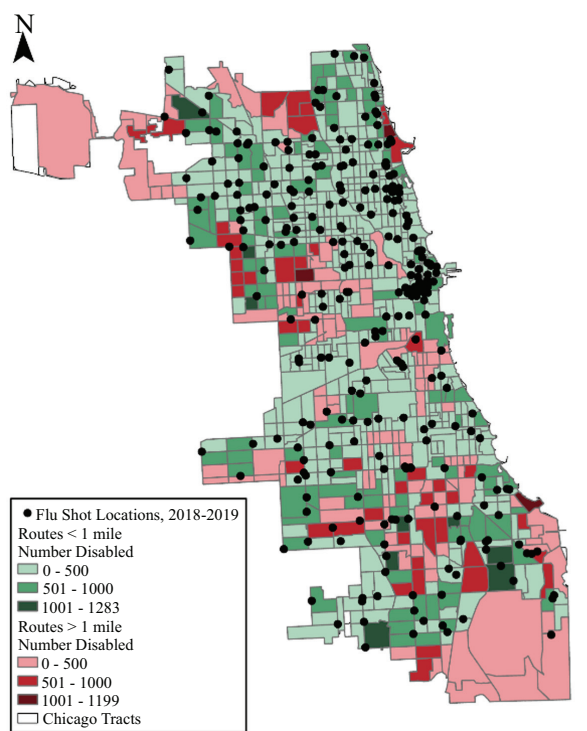

(a)

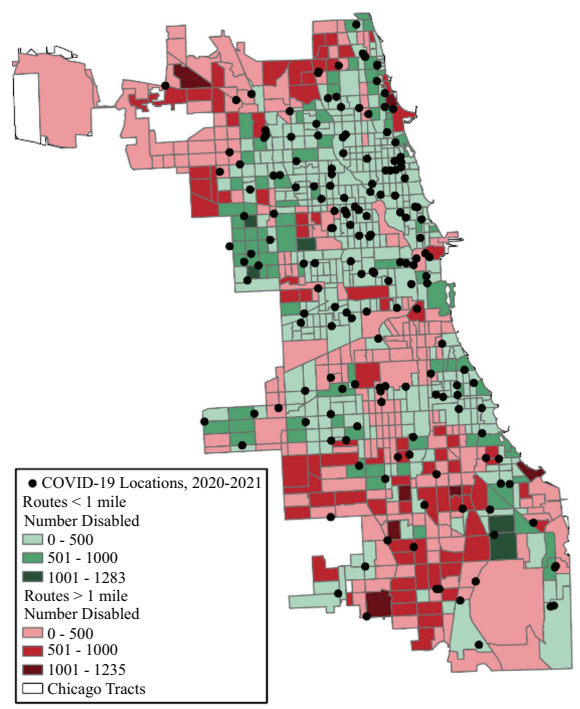

(c)

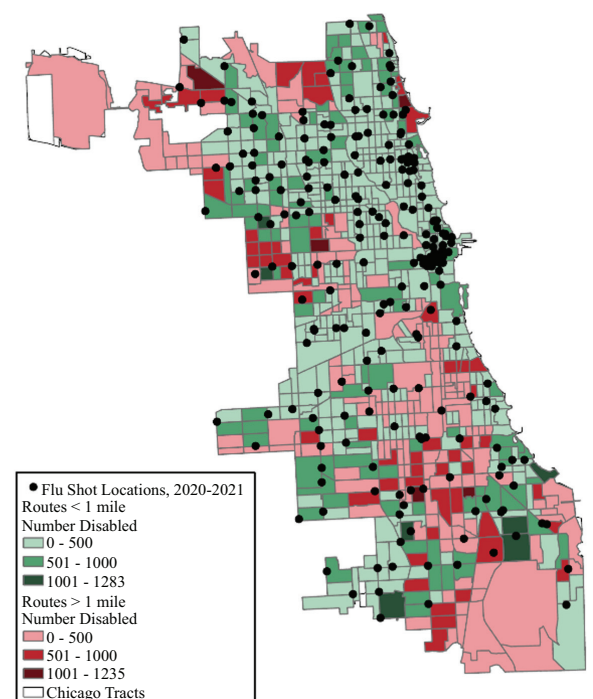

(b)

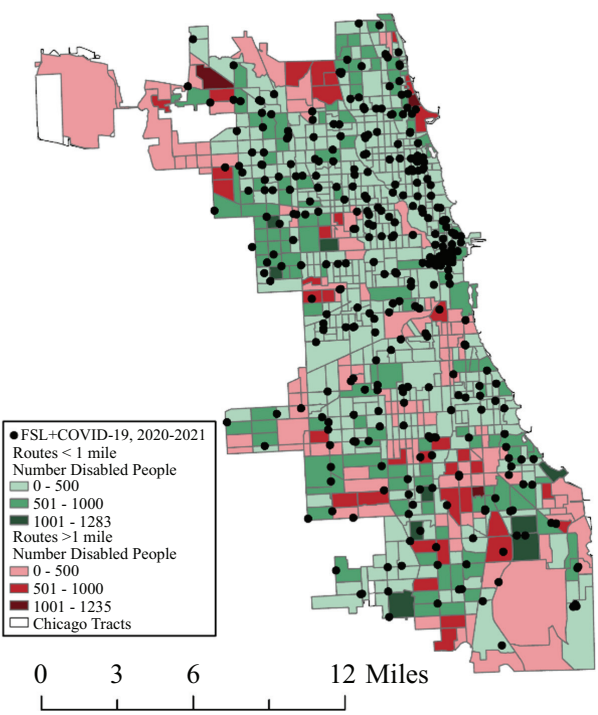

(d)

Source(s): Data from ACS 2018, CDC Social Vulnerability Index 2018, and Illinois Department of Public Health

$51.6 \%$ of the city's elderly located within one mile of a COVID-19 vaccination site. Figure 1D shows that by combining the 2020-2021 flu shot locations with COVID-19 flu shot locations, access improves from $51.6 \%$ of the elderly being within a mile to a vaccination site to $75.1 \%$. Minimizing the driving distance to the vaccination sites does not resolve the physical and 
socioeconomic barriers of access to the vaccination sites for people age 65 and older. Because there are other barriers besides travel distance make a situation unaffordable for older people, such as health insurance and access to a private vehicle.

Figure 2A shows that from 2018 to 2019, geographic flu vaccination access worsened for people with disabilities, particularly throughout south/central Chicago. Figure 2C's display of current COVID-19 vaccination sites, as of February 2021, suggests poor access, only $54.2 \%$ of the city's population with disabilities located within one mile of a COVID-19 vaccination site. Figure 2D shows that by combining the 2020-2021 flu shot locations with COVID-19 flu shot locations, access improves from $54.2 \%$ of people with disabilities being within a mile to a vaccination site to $76.8 \%$.

The decrease in flu vaccination sites from 2018-2019 to 2020-2021 is alarming, especially during a pandemic and particularly in areas of Chicago where minoritized communities are located. The lack of access to flu vaccination sites implies that access to COVID-19 vaccination sites will be similarly limited, which bears out based on COVID-19 vaccination site data from February 2021.

This study used publicly available data to better understand a rapidly developing health issue and how some of the most vulnerable are impacted. Flu and COVID-19 vaccination sites are appropriate locations to be distributing vaccines, yet this study is the first to combine them as a policy recommendation and shows geographic access increases when doing so.

Data and analysis were limited by the use of public data and could only analyze geographic access. Although both flu and COVID-19 vaccination data provided some information around cost/policy barriers to vaccine access, this portion of the data was unclear and incomplete and was not used in the final analysis. COVID-19 vaccination data is also continuing to change quickly, and so this analysis is only a snapshot of the vaccination landscape.

COVID-19 vaccination appointments are restricted to designated sites at no cost. For the underserved neighborhoods, registration phone lines and websites that can take hours to navigate, and lack of transportation make access to vaccination sites difficult. This study emphasizes the importance of COVID-19 vaccination sites in the nearest distance of the neighborhoods to reduce physical barriers of access for people with disabilities and aged 65 and older. Figure 2C displays the current nearest geographic access to the COVID-19 vaccination sites and illustrates the insufficient sites in the Southside of Chicago.

Future studies would collect more detailed data from flu and COVID-19 vaccination sites, such as cost, and other limitations. COVID-19 vaccinations are currently being distributed through several sites, including private practice healthcare providers, and temporary, groupspecific clinics. In the case of private practice healthcare providers, vaccines are available only to current patients of the provider. In the case of temporary, group-specific clinics, these are targeted events to groups falling within specific categories, such as certain essential worker groups. These temporary clinics may be held inconsistently throughout the vaccination period, and thus may not be recorded in public COVID-19 data sets. Future studies would collect this type of data retroactively from Chicago-based healthcare providers and organizations. Ideally, these studies would incorporate time as a barrier, showing access from both a geographic and temporal scale, and incorporate constraints that depend on the patient's work status or relationship to the provider.

To address healthcare access, it is more crucial than ever for policymakers and planners to work in concert with the data. The most socially vulnerable has long been the least often considered in healthcare access planning, and unfortunately, this seems to be the case as COVID-19 vaccination unfolds. However, the data and tools for analysis and for addressing these disparities exist. If a structured, data-based approach is used in concert with a social justice framework for vaccine and future booster distribution, spatiotemporal and authority constraints may be addressed before they become gaps in coverage.
Flu and

COVID-19

vaccination

sites 
JHR

36,5

866

\section{Conclusion}

Geographical methods contribute to studying the weaknesses and strengths of the health infrastructure. However, these methods are seldomly used in the realm of service provision for people with disabilities and people aged 65 and older with a higher risk of disability. The geographical method provided in this study highlights the importance of spatial analysis to study access disparity among people with disabilities and also explore opportunities in public health crises. Health service planners should design an integrative planning framework to align urban land-use models with health services. It is expected that these geographical approaches have a broader application in societies with disabled and older people where access to healthcare service needs an improvement.

Conflicts of Interest: None

\section{References}

1. Okoro CA, Hollis ND, Cyrus AC, Griffin-Blake S. Prevalence of disabilities and health care access by disability status and type among adults - United States, 2016. MMWR Morb. Mortal. Wkly. Rep. 2018; 67(32): 882-7. doi: 10.15585/mmwr.mm6732a3.

2. Roberts AW, Ogunwole SU, Blakeslee L, Rabe MA. The population 65 years and older in the United States. [updated 2018 Oct; cited 2021 Mar]. Available at: https://www.census.gov/library/ publications/2018/acs/acs-38.html.

3. Fitzpatrick AL, Powe NR, Cooper LS, Ives DG, Robbins JA. Barriers to health care access among the elderly and who perceives them. Am J Public Health. 2004; 94(10): 1788-94. doi: 10.2105/ajph.94.10.1788.

4. Houtenville A, Boege S. Annual report on people with disabilities in America. Durham, NH: University of New Hampshire; 2019. [cited $2021 \mathrm{Mar}$. Available at: https://www.iod.unh.edu.

5. Miller EA, Weissert WG. Geography still dictates Rx coverage for many near-poor seniors and disabled persons. J Aging Soc Policy. 2007; 19(1): 77-96. doi: 10.1300/J031v19n01_05.

6. Lebrasseur A, Fortin-Bédard N, Lettre J, Bussières EL, Best K, Boucher N, et al. Impact of COVID-19 on people with physical disabilities: a rapid review. Disabil Health J. 2021; 14(1): 101014. doi: 10.1016/j.dhjo.2020.101014.

7. Qato DM, Daviglus ML, Wilder J, Lee T, Qato D, Lambert B. 'Pharmacy deserts' are prevalent in Chicago's predominantly minority communities, raising medication access concerns. Health Aff (Millwood). 2014; 33(11): 1958-65. doi: 10.1377/hlthaff.2013.1397.

8. Illinois Department of Public Health [IDPH]. Flu shot location. [cited 2021 Mar]. Available at: https://www.dph.illinois.gov/topics-services/diseases-and-conditions/influenza.

9. Chicago Data Portal. COVID-19 vaccination sites. 2021. [cited 2021 Mar]. Available at: https://data. cityofchicago.org/Health-Human-Services/Flu-Shot-Locations-2014-Present-2015-2016-Season/er5x-2qdd.

10. American Community Survey [ACS]. Center of population by census tract. [cited 2021 Mar]. Available at: https://www.census.gov/geographies/reference-files/time-series/geo/centers-population.html.

11. Centers for Disease Control and Prevention [CDC]. Social vulnerability index database Illinois. [cited $2021 \mathrm{Mar}$. Available at: https://www.atsdr.cdc.gov/placeandhealth/svi/index.html.

12. Todd A, Copeland A, Husband A, Kasim A, Bambra C. The positive pharmacy care law: an arealevel analysis of the relationship between community pharmacy distribution, urbanity and social deprivation in England. BMJ Open. 2014; 4(8): e005764. doi: 10.1136/bmjopen-2014-005764.

\section{Corresponding author}

Aynaz Lotfata can be contacted at: alotfata@csu.edu

For instructions on how to order reprints of this article, please visit our website:

www.emeraldgrouppublishing.com/licensing/reprints.htm

Or contact us for further details: permissions@emeraldinsight.com 